\title{
O USO CLÍNICO DAS PENTAMIDINAS COM ESPECIAL REFERÊNCIA NAS LEISHMANIOSES.
}

\author{
Jackson Maurício L COSTA ${ }^{1}$
}

\begin{abstract}
RESUMO - O autor apresenta uma ampla revisão da literatura sobre as diamidinas aromáticas (Pentamidinas), dando ênfase à terapêutica das leishmanioses, considerando a possibilidade desta droga ser usada como segunda opção no tratamento das mesmas. Destaca aspectos relacionados à farmacodinâmica e efeitos adversos quando do uso clínico do referido medicamento.
\end{abstract}

Palavras Chaves: Pentamidinas, Leishmanioses, Uso Clínico.

Clinical Use of the Pentamidines with Special Reference to the Leishmaniases

ABSTRACT - The author presents an extensive literature review about aromatic diamidines (Pentamidines), giving emphasis therapeutic of the leishmaniasis, considering the possibility of this drug to be used as second line in the treatment of this disease. Detach aspects of pharmacodinamic and side effects in clinic use of this drug.

Key Words: Pentamidines, Leishmaniases, Clinical Use.

\section{INTRODUÇÃO}

A descoberta de atividade antiprotozoária no grupo das drogas diamidinicas, do qual as pentamidinas fazem parte, foi totalmente fortuita. Na década de 1920, desenvolveu-se metodologia capaz de manter tripanossomas vivos in vitro. Observou-se ser a glicose de fundamental importância para a sobrevida destes protozoários, fato este, sugerindo que agentes hipoglicemiantes tivessem efeito tripanossomicida.

A demonstração de que a diguanidina decametileno (synthalin), hipoglicemiante oral, possuía atividade terapêutica contra infecções murin causadas pelo Trypanosoma brucei, levaram YORK et al. (1940), a descrverem atividade tripanossomicida dosynthalin, juntamente as investigações iniciais sobre a ação das amidinas aromáticas em relação aos tripanossomas. Entre elas, a 4,4' diamidino difenoxipentano (pentamidina), como um dos 3 agentes descobertos, que demonstrava ação curativa contra o $T$. rhodesiense em camundongos infectados. Estas observações foram estendidas a trabalho de campo havendo demonstrações de que a $4,4^{\prime}$ diamidino diphenoxipropano (Propamidine) e a $4,4^{\prime}$ diamidino stilbene (Stilbamidine) eram eficazes contra os 3 agentes da tripanosomíase africana (T. rhodesiense, T. gambiense, T. congolense) SHOENBACH et al. (1948), KIRK et al. (1944), KING et al. $(1938,1944)$, SAUNDERS (1941).

1 Departamento de Patologia da Universidade Federal do Maranhão, disciplina Doenças Infecciosas e Parasitárias. Praca Madre Deus, $n^{\circ} 02$ (Pavilhão Pedagógico). CEP 65.025560 - São Luis (MA), Brasil. 
ELSON (1945), demonstrou que a propamidina em baixas concentrações possuia atividade fungistática contra $B$. dermatitides, $S$. schenkii e outros dermatófitos. Quanto às leishmanioses os primeiros relatos são de ADAMS (1941), tratando criança portadora de calazar com sucesso. Sendo que o isotionato de hidroxiestilbamidina (diisetionato de 2hidroxi-4-4'-diamidino estilbeno, foi a medicação de escolha por um longo periodo no tratamento do calazar na África Oriental (LOURIE et al., 1939), MANSON-BAHR (1953).

\section{Estrutura e Família}

As pentamidinas pertencem ao grupo das diamidinas aromáticas, compostos orgânicos que se caracterizam por possuirem uma cadeia alcano central inerte unida através de ligação éter ao grupo amidino polar terminal. Estruturalmente variam no comprimento da cadeia central, com a ligação molecular e na substituição da posição do carbono 2. A Figura 1, representa a fórmula estrutural do synthalin e das diamidinas aromáticas (Stilbamidine, 2-hydroxystilbamidine, propamidine e pentamidinas). CENTERS FOR DISEASE CONTROL (1984).

Em relação às pentamidinas, 2 sais, o isotionato di (Bhidroxietanosulfonato) e mesilato di (B-hidroximetilsulfonato) são comercialmente usados em humanos. MURDOCK et al. (1983). Nos Estados Unidos da America apenas o isotionato é comercializado, enquanto na Europa tem-se tanto o mesilato
(Lomidine) quanto $\mathrm{o}$ isotionato (Pentam, Pentacarinat). No Brasil a droga ainda não foi comercializada, sendo importada principalmente do laboratório May \& Baker Rhône Poulenc Group (Inglaterra). A Tabela 1, traz as diferenças entre o isotionato e o mesilato de pentamidina.

\section{Mecanismo de ação}

Segundo SANDS et al. (1985), o mecanisı ıo de ação das pentamidinas ainda não foi totalmente estabelecido, podendo diferir conforme o microorganismo contra os quais forem

Tabela 1. Diferenças existentes entre a pentamidina (Isotionato e Mesilato).

\begin{tabular}{|c|c|c|c|}
\hline \multirow{2}{*}{ Caracteristicas } & \multicolumn{2}{|c|}{ Isotionato Pentanidina * } & \multirow{2}{*}{$\begin{array}{c}\text { Mesilate } \\
\text { Pentumidina + }\end{array}$} \\
\hline & A & B & \\
\hline Aparencis frsica & $\begin{array}{l}\text { Tampáo seca/P6́ } \\
\text { Branco }\end{array}$ & Pó branco & Soluçào \\
\hline Conteódo ampola & 300 neisal & $200 \mathrm{mg} / \mathrm{sal}$ & $\begin{array}{l}120 \mathrm{ng} \text { base } / 3 \\
\text { id }\end{array}$ \\
\hline $\begin{array}{l}\text { Dose } \\
\text { (ne/kg/sinuples } \\
\text { injeça) }\end{array}$ & $4 \mathrm{mg} / \mathrm{kg} \mathrm{sel}$ & $4 \mathrm{mg} / \mathrm{sal}$ & $2,4 \mathrm{ng} / \mathrm{kg} / \mathrm{bare}$ \\
\hline $\begin{array}{l}\text { Dose móxims } \\
\text { diária }\end{array}$ & $300 \mathrm{mg} / \mathrm{sel}$ & $300 \mathrm{meg} / \mathrm{sal}$ & $180 \mathrm{mg} / \mathrm{buse}$ \\
\hline \multicolumn{2}{|c|}{$\begin{array}{l}\text { (A) Isotionato de pentamidina } \\
\text { Chemical (nil wauker). }\end{array}$} & produzida pelo labora & ratório Aldrich \\
\hline \multirow{2}{*}{\multicolumn{3}{|c|}{$\begin{array}{l}\text { (B) Isotionato de pentamidina produnido pelo Laboratório } \\
\text { Poulene Group. Inglaterra. } \\
\text { + Produzido pelo Laboratório Specia. França. }\end{array}$}} & May \& Rhòne \\
\hline & & & \\
\hline
\end{tabular}
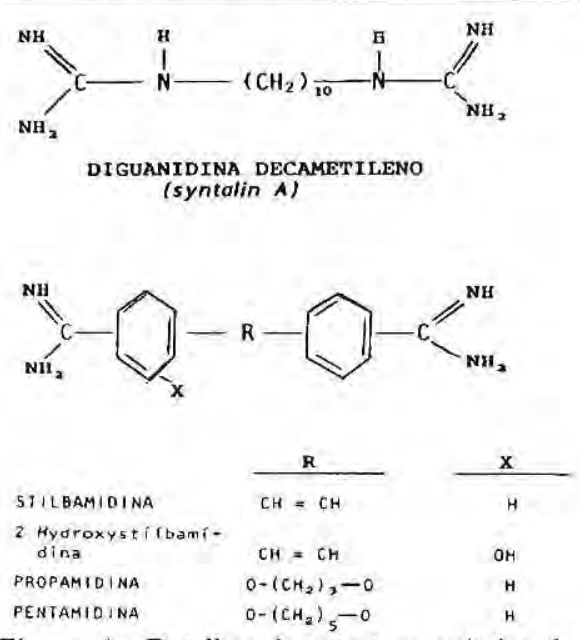

Figura 1. Detalhes da estrutura quimica do synthalin e das diamidinas aromáticas. 
utilizadas. "In vitro" foi observada ação das mesmas em bactérias como Staphylococcus aureus e Escherichia coli, tendo como provável mecanismo de ação a interferência no transporte de aminoácidos no metabolismo desses microorganismos. WARING (1965), GALE et al. (1967). Quanto aos tripanossomos, exercem ação tripanossomicida, através da interação destes compostos catiônicos com o DNA ou nucleotídeos e seus derivados, podendo ainda interferir na captação ou na função das poliaminas, inibição enzimática e da sintese de DNA, RNA. proteínas e fosfolipídeos, interferindo na respiração aeróbica e anaeróbica do microorganismo. MESHNICK et al. (1984), AL CHALABI et al. (1977).

Em relação ao Pneumocistis carinii, o mecanismo não está claro, contudo, a destruição do microorganismo não tem relação com a sintese do DNA ou com a inibição do metabolismo da glicose. As pentamidinas inibem a atividade da enzima dehidrofolato redutase quando testados contra extratos enzimáticos do figado e do rim de ratos infectados. AL CHALABI et al. (1977), PERSANTI (1980; 1981).

$\mathrm{Na}$ leishmaniose, estudos ultraestruturais em relação à infecção por $L$. tropica de um paciente antes e após terapêutica com pentamidina, mostrou modificações morfológicas no cinetoplasto, mitocôndria e ribossomas, com desintegração do cinetoplasto e uma rede filamentosa, alargamento mitocondrial com desaparecimento das cristas e uma diminuição do número de grânulos ribossomais. STECK et al. (1981), HENTZER et al. (1977). Na Leishmania amazonensis, em presença da pentamidina (in vitro), mostrou extensa ruptura da mitocôndria com fragmentação da membrana e crista e inibição da transformação de amastigota em promastigota. CROFT et al. (1982).

\section{Farmacocinética}

\section{Absorção, destino e excreção}

$\mathrm{O}$ isotionato de pentamidina é bem absorvido após administração parenteral. Poucos são os relatos sobre a farmacocinética da droga. estando mais concentrado em modelos animais. WAALKES et al. (1970), Com o advento da sindrome da imunodeficiência adquirida 9SIDA/ AIDS), a droga foi melhor estudada em humanos. Hoje, sabe-se que após uma única dose em pacientes com SIDA, a droga desaparece do plasma com meia vida aparente de 6 horas. Sendo eliminada lentamente na urina sob a forma inalterada, a sua depuração renal é de apenas $2 \%$ da depuração plasmática. CONTE et al. (1987), DONNELLY et al. (1988), SNAPPER et al. (1951)

$$
\text { WAALKES et al. (1970) }
$$
observaram que: após aplicação da dose standard de $4 \mathrm{mg} / \mathrm{kg} /$ peso do sal isotionato em humanos, os mesmos apresentaram niveis plasmáticos variáveis entre $0,3-1,4 \mathrm{ug} / \mathrm{ml}$. O pico máximo alcançado ocorreu aproximadamente 1 hora após injeção intramuscular. Com doses sucessivas houve 
pouca ou nenhuma elevação dos niveis da droga no soro. Aproximadamente 15 a $20 \%$ da dose diária era excretada na urina, com concentração urinária da droga aproximada de $20-25$ ug de pentamidina base $/ \mathrm{ml}$. Após cessar terapia ocorre diminuição dos níveis da droga podendo ser detectada na urina até 6 a 8 semanas.

DONNELLY et al. (1988), observaram em pacientes com SIDA, que após sucessivas doses por via parenteral, figado, rins, suprarenais, baço, continham as maiores concentrações da droga, enquanto o cérebro só apresentava diminutas quantidades. Os pulmões destes pacientes continham concentrações intermediárias. Deve-se obter concentrações pulmonares mais elevadas através da inalação de aerossóis de pentamidina na profilaxia ou no tratamento adjuvante da pneumonia por $P$. carinii de grau leve a moderado, a administração da droga por esta via resulta em pouca absorção sistêmica $\mathrm{e}$ toxicidade. MONTGOMEY et al. (1988, 1989), O'DOHERTY et al. (1988).

WALMAN et al. (1973), usando pendamidina aerossol em animais experimentais (ratos), observaram níveis significativos da droga no tecido pulmonar, mas nos rins, e no figado diminutos níveis foram encontrados.

Uso terapêutico, vias de administração e posologia

$\mathrm{O}$ isotionato de pentamidina é geralmente administrado por via parenteral, em injeções intramusculares ou infusões intravenosas lenta $(60$ minutos), em doses diárias ou dias alternados de $4 \mathrm{mg} / \mathrm{kg} /$ peso corporal. No tratamento da tripanosomiase africana de estágio inicial, deve-se administrar um curso de 10 injeções.

No tratamento da leishmaniose visceral tem sido utilizada com êxito em cursos de 12 a 15 doses. Um segundo tratamento, administrado depois de um intervalo de 1 a 2 semanas, pode ser necessário em áreas onde a infecção responde bem menos ao tratamento como foi observado no Quênia. BRYCESON et al. (1968, 1969, 1985), CHULAY et al. (1984).

JHA (1983), relatou sucesso no tratamento de 81 dos 82 casos de calazar resistentes aos antimoniais usando pentamidina no Norte Bihar, India. Na Leishmaniose cutânea, é considerada como droga de segunda escolha, usado em casos que não responderam aos antimoniais pentavalentes; temos observações em vários países africanos. NAVIN et al. (1984), BERMAN (1988). Nas Américas, poucos ensaios foram realizados tanto na forma cutânea, como na cutâneo mucosa, de um modo geral as respostas foram consideradas satisfatórias. TALHARI et al. (1985), SAMPAIO et al. (1988). PRADINAUD et al. (1985), SNAPPER (1952).

Na leishmaniose cutânea difusa (LCD), foi inicialmente utilizada por BRYCESON (1970) na Etiópia, quando tratou 33 pacientes com a forma mesilato de pentamidina, obtendo bons resultados quando comparados aos outros medicamentos utili- 
zados. No Brasil, MORAES (1993), utilizando $o$ isotionato de pentamidina na LCD, produzida por Leishmania amazonensis, obteve bons resultados iniciais, mas com o seguimento de 12 meses dos pacientes todos apresentaram recidivas das lesões.

Juntamente

com

o

sulfametoxazol-trimetropin, as pentamidinas constituem-se como droga de escolha do tratamento da pneumonia por $P$. carinii, a infecção oportunistica mais comum nos pacientes com SIDA. Os pacientes portadores de pneumonia por $P$. carinil, devem ser tratados com doses diárias de $4 \mathrm{mg} / \mathrm{kg} / \mathrm{via}$ intramuscular ou intravenosa durante 14 dias. A droga deve ser infundida por via intravenosa em 50 a $250 \mathrm{ml}$ de dextrose a $5 \%$, durante 60 minutos, a fim de evitar reações tóxicas imediatas. NAVIN et al. (1984), SANDS (1985). Se o tratamento for eficaz, a melhora clínica deve ser observada dentro de 4 a 6 dias após a $1^{\text {a }}$ injeção. Pode-se esperar uma elevada proporção de curas, dependendo da terapia de apoio e, se possível, da eliminação das condições predisponentes. O prognóstico é menos favorável em pacientes debilitados com alterações da imunidade ou com doença neoplásica, que podem necessitar mais de um curso terapêutico. A pentamidina pode ser ainda inalada sob a forma de aerossóis principalmente nos casos de SIDA. Ainda não foram estabelecidos esquemas posológicos ótimos, conseguindose profilaxia satisfatória mediante inalação mensal de $300 \mathrm{mg}$ da droga em solução aquosa nebulizando durante 30 a 45 minutos. ARMSTRONG (1988), GOLDEN (1989).

Em relação às micoses, observouse bons resultados com o uso da hidroxistilbamidine no tratamento da loporotricose quando localizada na pele ou na forma linfangitica, os quais não responderam à terapia com iodo. Foi usado ainda no tratamento da coccidioidomicose e nocardise com bons resultados. HARREL et al. (1954), BOCOBO et al. (1953), PEABODY et al. (1960).

\section{Toxicidade e efeitos colaterais}

Quando administrada a pentamidina, o clínico terá de acompanhar o paciente e observar complicações imediatas e tardias que poderäo advir. Segundo WALZER et al. (1974) e SANDS (1985), às complicações poderiam ser consideradas leves, como um simples mal estar, até mais graves como taquicardia venticular. A injeção intravenosa de pentamidina (e outras diamidinas), pode ser rapidamente seguida de reações alarmantes e por vezes perigosas. Incluem apnéia, taquicardia, tontura ou desmaio, cefaléia e vômitos. SANDS (1985), BRIGGAMAN (1977). É possivel que tais reações estejam relacionadas à súbita baixa da pressão sanguinea que ocorre após a administração intravenosa demasiadamente rápida, podendo ser decorrentes em parte, da liberação de histamina. Quando soluções de pentamidina não podem ser administradas lentamente por via intravenosa, a droga é bem tolerada após injeção, embora 
possa haver desenvolvimento de abcessos estéreis locais. O provável mecanismo seria a atuação da pentamidina como um hapteno, concentrando ácidos nucleicos no tecido local fazendo com que ocorra uma resposta imune local. NAVIM et al. (1984), SANDS (1985).

Acredita-se que as diamidinas aromáticas sejam consideradas libertadoras direta de histamina agindo nas células mães. Fica a dúvida sobre o porque de os antihistamínicos bloquearem parcialmente a reação. $\mathrm{O}$ isotionato de pentamidina não parece causar neuropatias tardias como as descritas frequentemente após tratamento com a estilbamidina. A pancreatite pode ocorrer, não sendo até o presente momento esclarecida; anormalidades no metabolismo da glicose podem ocorrer, havendo hipoglicemia seguida de hiperglicemia. O mecanismo da resposta bifásica ainda não está esclarecido, mas existem relatos de ser similar a ação do estreptozotocin e o N-3piridil-N-p-nitrourea, podendo induzir a uma citolise das células beta do pâncreas. $\mathrm{Na}$ fase inicial haveria liberação em excesso de insulina e hipoglicemia, seguida por uma diminuição da liberação de insulina, elevando os níveis glicêmicos, podendo evoluir para um quadro de diabetes melitus devido ao efeito tóxico sobre as células beta. WASKIN et al. (1988), BRYCESON (1969), KWAIME (1984).

Segundo BOUCHARD et al. (1982), o efeito diabetogênico pode ser cumulativo e dose dependente. Em muitos casos com deficit renal pré- existentes, OSEI et al. (1984), demonstraram "in vivo" que após aplicação da pentamidina as concentrações de glicose tendem a: 1) ocorrer aumento da concentração da glicose em poucos minutos, a qual é bloqueada pelos derivados da ergotamina, sendo atribuído a uma reação adrenérgica; 2) um subsequente periodo de hipoglicemia durante várias horas, sendo que após 5 a 17 horas ocorre hiperglicemia.

$O$ efeito diabetogênico da pentamidina pode ser retardado, tão logo quanto 5 a 8 meses após terapia em alguns pacientes. COLLINS et al. (1989), BELEHU et al. (1982), JHA et al. (1984).

A droga é nefrotóxica, mas, segundo SANDS et al. (1985), é reversivel, não sendo conhecidos até o presente momento o mecanismo provável de agressão renal. Concentrando-se nos rins, com aparente presença em toda área anatômica sendo conhecidas as interações com os ácidos nucleicos e inibição da enzima dehidrofolato redutase. Doses tóxicas de pentamidina produzem edema e degeneração dos tubulos renais em animais experimentais. Centers for Disease Control (1984), BOUCHARD et al. (1982). Pode ocorrer ainda leucopenia, neutropenia, anemia, trombocitopenia, alterações hepáticas, rush cutâneo, alopecia, hipocalcemia, diminuição dos níveis de folato $e$ indução de febre. MURPHEY et al. (1991), TONEKINS et al. (1972), SANDS et al. (1985), BOUCHARD et al. (1982). 
As alterações cardiacas com o uso das pentamidinas são por ordem defrequência: taquicardia, hipotensão, modificações no seguimento ST e onda T no eletrocardiograma. Existem poucos relatos de morte após uso da pentamidina. TOMKINS et al. (1972),

\section{Bibliografia Citada}

ADAMS, A. R. D. 1941, Studies in chemotheraphy. XXVI. A case of indian Kala-azar treated with 4:4'-diamidino diphenoxypentane. Annals Tropical Medicine Parasitology, 35:53-54.

ALCHALABI, K.; GUTTERRIDGE, W. E. 1977. Presence and properties of thymidylate sinthetase in trypanosomatids. Biochemestry Biophysical Acta, 481:71-79.

ARMSTRONG, D.; BERNARD, E. 1988. Aerosol pentamidine. Annals of Internal Medicine, 109:852-854

BELEHIJ, A., NAAFS, B. 1982. Diabetes Mellitus associated with pentamidine mesylate (letter), Lancet(1):1463:1464.

BERMAN, J. D. 1988. Chemotheraphy for leishmaniasis: biochemical mechanisms. clinical efficacy, and future strategies. Reviews of Infectwous Diseases, 10:560586.

BOCOBO, F; CURTIS, A; HANREL. E. R. 1953. In vitro fungistatic activity of stilbamidine, propamidine, pentamidine and diethylstilbestrol. Joumal Investigation Dermatology, 21:149.

BOUCHARD, P., SAL, P., REACH, G.; CANBARRERE, I., GANEVAL, D.; ASSAN, R. 1982. Diabetes mellitus following pentamidine induced hypoglycemia in humans. Diabetes, $31: 40-4$

BRIGGMAN, R A. 1977. The aromatic Diamidines. International Journal of Dermatology, 16(3):155-162.
BRYCESON, A. 1968. Pentamidine-induced diabetes mellitus. East African Medical Journal, 45:110-117.

BRYCESON, A.; WOODSTOCK, L. 1969. The cumulative effect of pentamidine dimethanesulphonate on the blood sugar. East African Medical Journal, 46(3):170-173.

BRYCESON, A. D. M. 1970. Diffuse cutaneous leishmaniasis in Ethiopia: II Treatment. Transactions of the Royal Society of Tropical Medicine, 64(3):369-379.

BRYCESON, A. D. M.; CHULAY, J. D.; MUGAMBI, M.; WERE, J. B.; GACHIHI, G.; CHUNGE, C. N; MIGAI, R; BHATT, S. M.: HOM:; SPENCER, H. C.; MEME, J.; ANABE WANI, G. 1985. Visceral leishmaniais unresponsive to antimonial drugs. II. Response to high dosage sodiun stibogluconate or prolonged treatment with pentamidine. Transaction of the Royal Society of Tropical Medicine and Hygiene. 79:705-714.

CENTERS FOR DISEASE CONTROL US. 1984. Manufectered pentamidine isethionate eleared for investigational use. MMWR, 33:270.

CENTERS FOR DISEASE CONTROL. 1984. Severe neutropenia during pentamidine treatement of Pneumocystis carinit pneumonia in patients with acquired immubodeficienty syndrome. New York City. MMWR, 31:40-45

COLLINS, R. J.; PIEN, F, D.; HOUK, J. H. 1989. Case report: insulin-dependent diabetes mellitus associated with pentamidine. American Journal of Medicine and Science, 297:174-175.

CONTE, J. E., UPTON. JR., R. A.; LEN, E. T. 1984. Pentamidine pharmacokinetics in patients with AIDS with impaired renal function. Journal Infectuous Diseaes, 156:885-890.

CHULAY, J. D., MANSON-BAHR. P. E. C. 1984. Visceral Leismaniasis (KALA- 
AZAR). In: STRICEAND GT, (ed.) Hunter's Tropical Medicine, 6 th. ed. Philadelphia: WB Saunders. p.578-585.

CROFT, S. L.; BRAZIL, R. P. 1982. Effect of pentamidine isethionate on the ultrastructural and morphology of Leishmania mexicana amazonensis in vitro. Annals of Tropical Medicine and Parasitology, 76(1):37-43.

DONNELLY, H; BERNARD. E. M ; ROTHOKOTTER, H: GOLD, J. W, M. ARMSTRONG, D, 1988. Distribution of pentamidine in patients with AIDS Journal of Infectuous Diseases, 157:985-989.

ELSON, W. 1945. Antibacterial and fungistatic properties of propamidine. Journal of Intectuous Diseases, 76:193.

GALE, E. F.; FOLKES, J. P. 1967. Actions of pentamidine on the metabolism of Staphylococcus aureus. Biochemistry Biophisics Acta, 144:467-470.

GOLDEN, J. A.; CHERNOFT, D,; HOLLANIDER, H.; FEIGAL, D.: CONTE, J

E. 1989. Prevention of Pneumocystis carinnii pneumonia by inhaled pentamidine, Lancet, 1:654-657

HARREL, E. R.; BOCOBO, F C.; CURTIS, A. C. 1954. Sporotricosis sucessfully treated with stilbamidine. Archives of International Medicine, 93:162.

HENTZER, B.; KOBAYASI, T. 1977. The ultrastructural changes of leishmania tropica after treatment with pentamidine. Annals of Tropical Medicine and Parasitology, 71(2):157-166.

JHA, T. K. 1983. Evaluation of doamidine compound (pentamidine isethionate) in the treatment of resistent cases of KALA-AZAR occurring in North Bihar, India. Transactions of the Royal Society of Tropical Medicine and Hygiene, $77(3): 167-170$.

JHA, T. K., SHARMA, V. K. 1984. Pentamidine-induced diabetes mellitus. Transactions of the Royal Society of Tropical Medicine and Hygiene, 78:252253.
KING, H.; LOURIE, E. M.; YORKE, W. 1938. Studies in ehemotherapy. XIX. Further report on new trypanocidal substances. Annals of Tropical Medicine and Parasitology, 32:177-192.

KIRK, R; HENRY, A. 1944. Observations on the toxicity of stilbamidine. Annals of Tropical Medicine and Parasitology. 38:99.

KWAME, O; FALKO, J. M; NELSON, K. P; STEPHENS, R. 1984. Diabetogenic effect of pent.midine. In vitro and in vivo studies in a patient with malignant insulinoma. American Journal of Medicine. $7^{n}+16$.

LOURIE, E. M: ' ORKE, W. 1939. Studies in chemolherapy. XXI. The trypanocidal action of Certain aromatic diamidines. Annals of Tropical Medicine Parasitology. 33:289-304.

MANSON-BAHR, P. E, C. 1959 East african KALA-AZAR with special referenceto the pathology, prophylaxis and treatment. Transactions of the Royal Society of Tropical Medicine and Hygiene, 53:123-137.

MONTGOMERY, A. B.; DEBS, R. J; LUCE. J. M. CORKERY, K. J; TURNER, I: BRUNNETTE, E. N ; I.IN, E. T.; HOPEWELL. P. C. 1988. Selective delivery of pentamidine to the lung by aerosol. American Review Respiratory Disease, 137:477-478

Aerosolized pentamidne as second line therapy in patients with AIDS an Pneumocistis carinii pneumonia. Chest. 95:747-750.

MORAES, M. S. C. 1993. Ensaio terapêtico com lsotionato de Pentamidina (Pertacarinat) em pacientes portadores de $L C D$ no estado do Maranhão-Brasil. Monografia de conclusão do curso de Medicina. 101p.

MURDOCK, J. K.; KEYSTONE, J. S. 1983. Two forms of pentamidine. Canadian Medical Association Joumal, 128(1):508.

MURPHEY, S. A.; JOSEPHS, A. S. 19.81. Acute pancreatitis associated with 
pentamidine therapy. Archives Internal Medicine, 141:56-58.

NAVIN, T, R. \& FONTAINE, R. E. 1984. Introvenous versus intramuscular administration of pentamidine. New England Journal of Medicine, 311:17011702.

O'DOHERTY, M. J; THOMAS, S ; PAGE, C.; BARBOW, D.; BRADBEERL, C.; NUNAN, T. O,; BATEMANI, N. T. 1988. Differences in relative efficiency of nebulisers for pentamidine administration. Lancet, 2:1283-1286.

OSEI, K.; FALKO, J. M; NELSON, K. P; STEPHENS, R. 1984. Diabetogenic effect of pentamidine. American Journal of Medicine, 77:41-46.

PEABODY, J.; SEABURY, J. 1960. Actinomycosts and Nocardiosis. American Journal of Medicine, 28:99.

PERSONTI, E. 1980. In vitro effects of antiprotozoan drugs and immune serum on Pneumocistis carinii in vitro. Journal of infectuous Diseases, 141:775-780.

PERSONTI, E; COX, C. 1981. Metabolic and synthetic activities of Pneumocystis cardini in vitro. Infection immunity. 34:908-914.

PRADINAUD, R.; GIRARDEU, I.; SAINTEMARIE, D. 1985. Pentamidina. Excelente terapêutica da leishmaniose cutânea, Esquema de tratamento idealizado na Guiana Francesa em dose única. Anais Brasileiros de Dermatologia, 60(6):385-387.

SAMPAIO, R. N. R; SOARES, S. K. P; ROSA, A. C.; NETTO, E. M; MAGALHÃES, A. V.: MARSDEN, P

D. 1988. Tratamento com pentamidina de seis casos da forma mucosa da leishmaniose tegumentar. Anais Brasileiros de Dermatologia, 63(6):439-442.

SANDS, M.; KRON, M. A.; BROWN, R. B. 1985. Pentamidine: a review. Reviews of Infectuous Diseases, 41:187-194.

SAUNDERS, G. F. T. 194I. Preliminary report on the treatment of sleeping sickness by $4: 44^{\prime}$ diamidino diphenoxypentane.
Annals Tropical Medicine and Parasitology, 35:169-174.

CHOENBACH, E. B.; GREENSPAN, E. M. 1948. The pharmacology, mode of action and therapeutic potentialities of Stilbamidine, pentamidine, propamidine and other aromatic diamidines. A Review. Medicine (Baltimore), 27:327-377.

SNAPPER, I.; LIEBEN, R.; GREENSPAN, E.; SCHNEID, D. 1951. Determination of stilbamidine and a hydroxsxtilbamildine, deposited in parenchymatous organs and tumours. Cancer, 4:1246.

SNAPPER, J 1952. American mucocutaneous leishmaniasis sucessfully treated with 2-hydroxystilbamidine. American Journal of Medicine, 13:655.

STECK, E. A.; KINNAMON, K. E.; RANE, D. S.; HANSON, W. L. 1981. Leishmania donovani. Plasmodium berghei, Trypanosoma hodesiense: Antiprotozoal effects of some amidine types. Experimental Parasitology. 52:404-413.

TALHARI, S.; SARDINHA, J. C. G: SCHETTINNI, A. P. M.; ARIAS, J.; NAIFF, R. D. 1985. Tratamento da Leishmaniose tegumentar americana. Resultados preliminares com a pertamidina. Anais Brasileiros de Dermatologia, 60(6):361-364.

TOMKINS, A; BRYCESON, A. 1972. Ocular leishmaniais and pentamidine diabetes. Transactions of the Royal Society of Tropical Medicine and Hygiene, 66:948-949.

WAALKES, T. P; DE VITA, B. T. 1970. The determination of pentamidini $(4,4$ 'diamidinophenoxypentane) in plasma, urine, and tissue Journal of Laboratory Clinical Medicine, 75:871-878.

WAALKES, T. P; DENHEM, C.; DE VITA, V. T. 1970 . Pentamidine: Clinical pharmacologic correlation in man and mice Clinical Pharmacological Therapy, 11:505-512.

WALMAN, R.; PEARCE, D.; MARTIN, R. 1973. Pentamidine isethionate levels in 
lungs, livers and kidneys of rats after aerosol or intramuscular administration. American Review Respiratory Disease, 108:1004-1006.

WARING, M. J. 1965. The effects os antimicrobial agents on ribonucleic acid polymerase. Molecular Pharmacology. 1:1-13.

WASKIN, H., STEHR-GREEN, J. K: HELMICK, C. G: SATTLER, F, P. 1988. Risk factors for hypoglycemia associated with pentamidine therapy for pneumocystis pneumonia. Journal of American Medical dcademy. 260:345347.
WESTERN, K. A.; PERERA, D. R.;SCHULT, M. G. 1970, Pentamidine isethionate in the treatment of Pneumocistis carinii pneumonia. Annals of Internal Medicine, 73:695-702

YORKE, W. 1941. Recent work on the chemotherapy of protozoal infections. Transactions of the Royal Society of Tropical Medicine and Hygiene, 33:463472. 